\title{
Delayed healing and induction of secretory leukocyte protease inhibitor in polycystic ovary syndrome rat skin wounds
}

\author{
SOON-JEONG JEONG ${ }^{1 *}$, SUNG-SHIN KIM $^{2 *}$, CHUN-SIK BAE $^{3}$, JIN-JU PARK $^{1}$, BAIK-DONG CHOI ${ }^{1}$, \\ GUANLIN WANG ${ }^{1}$, MYUNG-JU JUNG ${ }^{1}$, HYUN-SUN JANG ${ }^{2}$, BYUNG-OCK KIM ${ }^{2}$, \\ DO-SEON LIM ${ }^{4}$, YOUNG-SIK CHO ${ }^{5}$ and MOON-JIN JEONG ${ }^{1}$
}

\begin{abstract}
Departments of ${ }^{1}$ Oral Histology and Developmental Biology, and ${ }^{2}$ Periodontology, School of Dentistry, Chosun University, Gwangju 501-759; ${ }^{3}$ College of Veterinary Medicine, Animal Medical Institute, Chonnam National University, Gwangju 500-757; ${ }^{4}$ Department of Dental Hygiene, Eulji University, Seongnam, Gyeonggi-do 461-713;

${ }^{5}$ Department of Dental Hygiene, Namseoul University, Chunan 331-707, Republic of Korea
\end{abstract}

Received July 28, 2011; Accepted September 2, 2011

DOI: $10.3892 /$ ijmm.2011.816

\begin{abstract}
Secretory leukocyte protease inhibitor (SLPI) and estrogen promote wound healing through a decrease in the excessive inflammatory response, accelerating re-epithelialization and increasing the amount of collagen deposition. The excessive administration of estradiol valerate (EV) using hormonal therapy decreases the concentration of estrogen abruptly and induces the polycystic ovary syndrome (PCOS). In this study, the PCOS rat skin wound area was wider than that of the normal groups and the rate of keratinocyte migration in PCOS was lower than the normal group. The numbers of inflammatory cells and macrophages recruited in the PCOS group were larger than that of the normal group. More collagen was deposited in the healing area of the normal group than in the PCOS group. The level of SLPI expression was higher in the PCOS group than the normal group after wounding, with the exception of the epithelium. On the other hand, mRNA and protein expression levels of transforming growth factor- $\beta 1$ (TGF- $\beta 1$ ) were lower in the PCOS group than in the normal group. Matrix metalloproteinase-2 (MMP-2) and MMP-9 levels in the PCOS group were significantly lower than that of the normal group. Therefore, increased SLPI in PCOS skin wounds may help prevent an excessive inflammatory response and aberrant collagen deposition but not are sufficient to accelerate PCOS skin wound healing, suggesting that SLPI may act as a local rather than a systemic modulating molecule in PCOS rat skin wounds.
\end{abstract}

Correspondence to: Professor Moon-Jin Jeong, Department of Oral Histology and Developmental Biology, School of Dentistry, Chosun University, 375 Seo-Suk Dong, Gwangju 501-759, Republic of Korea E-mail: mjjeong@chosun.ac.kr

*Contributed equally

Key words: secretory leukocyte protease inhibitor, estrogen, polycystic ovary syndrome, wound healing, rat skin

\section{Introduction}

Cutaneous wound healing is a complex and highly coordinated process. In general, cutaneous wound healing is divided into three major phases, hemostasis and inflammation, re-epithelialization and the formation of granulation tissue, and matrix remodeling. These phases are histologically and functionally distinct but they overlap temporally, and complete healing requires the interactive, carefully orchestrated communication of many cell types such as inflammatory cells, fibroblasts and keratinocytes in distinct tissue compartments, which induce a change in the microenvironments (1).

Polycystic ovary syndrome (PCOS), which is one of the most common causes of anovulation in women of reproductive age, is a complex endocrine and metabolic disorder. The antral-follicle, which produces androgen, is frequently observed in the PCOS ovary instead of the dominant preovulatory follicles (2-4). PCOS is a hormonal imbalance condition that affects the endocrine system and gives rise to complications, such as obesity and diabetes. The skin of women with PCOS shows abnormalities related to excess androgen, such as hirsutism, acne and alopecia (5). Estradiol valerate (EV) is one of the substitute molecules for estrogens used in menopausal women. The excessive administration of EV, however, induces PCOS following various side effects, such as breast tenderness, headache and plantar swelling (6). In addition, an injection of EV decreases the circulating estrogen concentration abruptly (2). This hormonal imbalance delays the recovery and inhibition of the healing in chronic wounds, such as psoriasis that occurs in women (7).

Sex-hormone plays an important role in the wound healing process and has a variety of functions during the wound healing process, such as a mitogenic effect on keratinocytes, increasing the rate of re-epithelialization. In addition, estrogen increases the formation of granulation tissue and collagen deposition by promoting secretion of transforming growth factor- $\beta 1$ (TGF- $\beta 1$ ) from fibroblasts in the dermis (8-10). A local injection or systemic treatment of estrogen was reported to accelerate healing due to a shortened period of the inflammatory response in wounds of men and elderly women $(8,11)$. 
Human secretory leukocyte protease inhibitor (SLPI) exists as a non-glycosylated, hydrophobic, cationic $12 \mathrm{kDa}$ protein that was originally isolated from human parotid gland secretions. The protein is composed of 130 amino acids. The $\mathrm{N}$-terminal domain of SLPI has an antibiotic function and the C-terminal is the docking site for protease inhibition $(12,13)$. SLPI has been described as an anti-inflammatory factor during the early inflammatory response in odontoblasts (14) and plays a role not only to protect the tissues from proteases (4) but also to promote wound healing, cell proliferation and migration, and inhibition of HIV infections. It has also been described as having anti-bacterial and anti-fungal activity (15-17).

PCOS accompanied by complicating disease may delay skin wound healing. Recently, it was reported that skin wound healing was delayed significantly in ovariectomized (OVX) mice to block the secretion of estrogen from the ovary (18). SLPI promotes cutaneous and oral mucosal wound healing $(19,20)$, and estrogen regulates SLPI expression $(21,22)$. Although SLPI and estrogen show a functional relationship in wound healing, the role and expression of SLPI in PCOS skin wound healing is unclear. Therefore, this study examined the expression and function of SLPI in the cutaneous wound healing of PCOS rats with a hormonal imbalance.

\section{Materials and methods}

Induction of the PCOS condition. Seven week old virgin adult female Sprague Dawley strain rats (Samtako Bio, Korea), weighing 190-210 g, were used. Animals showing at least two regular 4-day sexual cycles were selected for the experiments. The PCOS was induced by the administration of EV (Sigma-Aldrich, USA) as a single intramuscular injection (4 mg). The 60 days interval was chosen as the last time point because it corresponds to the time when the cysts become clearly established (3). The temperature and humidity was maintained at $23 \pm 2^{\circ} \mathrm{C}$ and $60 \pm 10 \%$, respectively. The animals were kept on a $12 \mathrm{~h} \mathrm{light,} 12 \mathrm{~h}$ dark photoperiod and allowed free access to pelleted rat chow and tap water. All animal studies were approved by the 'Institutional Animal Care and Use Committees' at Chosun University and animal care was carried out in SPF level systems according to the 'Guide for the Care and Use of Laboratory Animals'.

Estrogen RIA. Estrogen levels in the plasma were measured by radioimmunoassay (RIA). The general assay procedure was adapted from a previous publication (23). Labeled estradiol-17 $\beta$ [estradiol-6-(O-carboxymethyl)] oximino-[2-( $\left.\left.{ }^{125} \mathrm{I}\right)\right]$ iodohistamine) was obtained from Amersham Biosciences. The radioactivity of each sample was quantified using a gamma counter (Perkin-Elmer Life Sciences, Auto Gamma 5005). Routinely, two sets of standard (5-2,000 pg) were included in each assay. Estrogen concentration was calculated with the RiaSmart program (Perkin-Elmer Life Sciences). Between and within assay coefficients of variation (CVs) were 9.4 and $9.2 \%$, respectively. The lower limits of assay sensitivity were $2.5 \mathrm{pg}$.

Induction of dorsal skin wound. A total of 28 rats were divided equally into the normal and PCOS group, respectively. The rats were anesthetized with a mixing solution of $0.2 \mathrm{ml} / 100 \mathrm{~g}$ ketamine (Yuhan, Korea) and $0.08 \mathrm{ml} / 100 \mathrm{~g}$ Rompun (Bayer,
Korea) as a single intramuscular injection. The dorsal hair of the rats was shaved and cleaned with ethanol. Six equidistant $(1 \mathrm{~cm})$ full-thickness incisional wounds were made using a surgical blade underlying the panniculus carnosus muscle. After wounding, the rats were sacrificed and the wound skin was excised on Days 1, 3, 5 and 7, respectively. No wound tissue of the dorsal skin with the normal and PCOS group was used as the control.

Histology and histomorphometric analysis. Excised skin wounds were fixed in $10 \%$ buffered neutral formalin and incubated overnight at $4^{\circ} \mathrm{C}$. The tissues were washed in $1 \mathrm{X}$ PBS for $2 \mathrm{~h}$ and dehydrated by sequential washing in 70,80 , 90, 100 I, 100 II, 100 III and finally 100\% IV ethanol. The clearing process was performed using xylene, and the tissues were embedded in paraffin. The paraffin blocks were cut into $6 \mu \mathrm{m}$ thick sections using a Motorized Rotary microtome MT990 (RMC Products, USA) and the sections were attached to a $37^{\circ} \mathrm{C}$ slide warmer overnight after placing them onto a slide glass coated with 3-(trimethoxysilyl) propyl methacrylate (Sigma, USA). Hematoxylin and eosin (H\&E) staining was performed to observe the difference in the histological changes and to measure the wound area. The inflammatory cells were counted in Giemsa stained tissues. Masson's Trichrome (MT) and Picrosirius Red staining were carried out to assess collagen deposition during matrix remodeling. The wound area was determined by measuring the area between the wound edges below the scab. Keratinocyte migration was measured as the length of the epidermal tongues from both wound edges and the data are expressed as the percentage wound closure (distance of migrated keratinocyte from the wound edge/total wound width x100). The inflammatory cells were counted in three distinct areas (left, middle and right wound area). Wound contracture was measured from the distance between the wound edge and invaded dermis (sum of distance of contracted dermis from wound edges/total wound width $\mathrm{x} 100$ ). The stained tissues were observed by optical microscopy (Carl Zeiss, Germany) and the tissues stained with Picrosirius Red were observed by polarizing microscopy (Carl Zeiss). Histomorphological analysis was performed using the AxioVision LE release 4.6 (Carl Zeiss) program.

Extraction of total-RNA and reverse transcription and polymerase chain reaction. The tissues were ground after being deep-frozen in liquid $\mathrm{NO}_{2}$ and total-RNA was extracted with TRI Reagent (MRC Inc., USA) according to the manufacturer's instructions. A $1 \mu \mathrm{g}$ sample of the total-RNA was used to synthesize the complementary DNA (cDNA). The synthesis of cDNA was performed with AccuPower RT PreMix (Bioneer, Korea). The PCR reaction was carried out in a thermal cycler (Takara, Japan) after adding a $1 \mu \mathrm{l}$ of cDNA to the AccuPower PCR PreMix (Bioneer). The following primers (Bioneer) were used for RT-PCR analysis: i) rat SLPI forward, 5'-ATC AAA ATC GGA GCC TGC CC-3' and reverse, 5'-TGG CCG TCA TTC TGG CAC TT-3'; ii) rat TGF- $\beta 1$ forward, $5^{\prime}$-CTG TCC AAA CTA AGG CTC GC-3' and reverse, 5'-AGA CAG CCA CTC AGG CGT A-3'; iii) rat glyceraldehyde 3-phosphate dehydrogenase (GAPDH) forward, 5'-CCA TGG AGA AGG CTG GG-3' and reverse, 5'-CAA AGT TGT CAT GGA TGA CC-3'. GAPDH was used as the internal control for RT-PCR. The 
PCR condition for SLPI was as follows: initial denaturation for $5 \mathrm{~min}$ at $94^{\circ} \mathrm{C}$, followed by 32 cycles of denaturation at $94^{\circ} \mathrm{C}$ for $40 \mathrm{sec}$, annealing at $66^{\circ} \mathrm{C} 40 \mathrm{sec}$ and extension at $72^{\circ} \mathrm{C}$ for $35 \mathrm{sec}$. RT-PCR for TGF- $\beta 1$ was as follows: initial denaturation for $5 \mathrm{~min}$ at $94^{\circ} \mathrm{C}$, followed by 35 cycles of denaturation at $94^{\circ} \mathrm{C}$ for $30 \mathrm{sec}$, annealing at $60^{\circ} \mathrm{C} 30 \mathrm{sec}$ and extension at $72^{\circ} \mathrm{C}$ for $30 \mathrm{sec}$. RT-PCR for GAPDH was as follows: $5 \mathrm{~min}$ at $94^{\circ} \mathrm{C}, 30$ cycles at $94^{\circ} \mathrm{C}$ for $30 \mathrm{sec}, 30 \mathrm{sec}$ at $56^{\circ} \mathrm{C}, 30 \mathrm{sec}$ at $72^{\circ} \mathrm{C}$. After the last cycle, all samples were incubated for an additional $5 \mathrm{~min}$ at $72^{\circ} \mathrm{C}$. The products were electrophoresed on a $1.5 \%$ agarose gel buffered with $0.5 \mathrm{X}$ Tris-Borate-EDTA and stained with ethidium bromide after amplification. The staining bands were visualized by Gel-Doc (Bio-Rad Laboratories, USA). The primer sets specifically recognized only the genes of interest, as indicated by the amplification of a single band of the expected size (570 bp for SLPI, $501 \mathrm{bp}$ for TGF- $\beta 1$ and $199 \mathrm{bp}$ GAPDH) according to the nucleotide sequence of SLPI (GenBank no. NM_003064), TGF- $\beta 1$ (GenBank no. NM_021578.2) and GAPDH (GenBank no. M33197.1). The intensity of the bands was measured using a Science Lab Image Gauge (FujiFilm, Japan).

Western blotting. Western blotting was performed to examine the expression of SLPI, TGF- $\beta 1$ and $\beta$-actin protein. The tissues were ground after being deep-frozen by liquid $\mathrm{NO}_{2}$ and incubated with NP-40 lysis buffer (150 mM NaCl, $1 \%$ NP-40, $50 \mathrm{mM}$ Tris- $\mathrm{HCl}$ ( $\mathrm{pH}$ 7.4), $2 \mathrm{mM} \mathrm{Na}_{3} \mathrm{VO}_{4}, 2 \mathrm{mM} \mathrm{Na} \mathrm{P}_{2} \mathrm{O}_{7}$, $50 \mathrm{mM}$ NaF, $2 \mathrm{mM}$ EDTA (pH 7.4) $0.1 \mu \mathrm{g} / \mathrm{ml}$, leupeptin and $1 \mu \mathrm{g} / \mathrm{ml}$ aprotinin) on ice for $30 \mathrm{~min}$. After protein extraction, the concentration in $30 \mu \mathrm{g}$ was determined using a Dc Protein Assay kit (Bio-Rad Laboratories) and electrophoresed onto 10-15\% SDS polyacrylamide gels. After electrophoresis, the protein was transferred to a nitrocellulose membrane and blocked with $5 \%$ non-fat dry milk for $1 \mathrm{~h}$ at room temperature. The membrane was blotted with 1:500 of anti-rabbit SLPI (14), 1:1,000 of anti-rabbit TGF- $\beta 1$ (Santa Cruz Biotechnology, Santa Cruz, USA) and 1:2,500 of anti-mouse $\beta$-actin (Sigma) for $16 \mathrm{~h}$ at $4^{\circ} \mathrm{C}$. After washing, the membrane was blotted with 1:5,000-1:10,000 of HRP-conjugated goat anti-rabbit or mouse-IgG (Santa Cruz Biotechnology) and developed using chemiluminescence film after being treated with an ECL solution (Amersham Pharmacia Biotech). The size of the SLPI, TGF- $\beta 1$ and $\beta$-actin bands was a 12,25 and $42 \mathrm{kDa}$, respectively. The density of the expressed bands was measured using a Science Lab Image Gauge (FujiFilm).

Immunohistochemistry. The sample was deparaffinized sequentially in xylene I, II and III for 5 min each, and hydrated sequentially in 100, 90, 80 and $70 \%$ ethanol for $5 \mathrm{~min}$. The sample was washed three times in $1 \mathrm{X}$ PBS for $5 \mathrm{~min}$ and primary blocked with $0.6 \% \mathrm{H}_{2} \mathrm{O}_{2}$ in methanol for $20 \mathrm{~min}$ at room temperature. Normal goat serum (15 $\mu 1$ in $1 \mathrm{ml}$ PBS, Vector Lab, USA) was used for secondary blocking. After washing, the sample was incubated with anti-rabbit SLPI (1:500) (14) or anti-mouse Mac-3 (1:250, BD Biosciences Phamingen, USA) diluted in fresh normal goat serum for $16 \mathrm{~h}$ at $4^{\circ} \mathrm{C}$. The negative control sample was not incubated with primary antibodies. Goat-anti-rabbit or mouse IgG (1:200, Vector Lab) were used as the secondary antibodies for $20 \mathrm{~min}$ at room temperature. After washing twice with PBS for $10 \mathrm{~min}$, the sample was incubated with an $\mathrm{ABC}$ reagent for $20 \mathrm{~min}$ and developed for $1 \mathrm{~min}$ and $40 \mathrm{sec}$ using the $0.05 \%$ DAB (diaminobenzidine tetrahydrochloride, Vector Lab). After washing twice with $\mathrm{ddH}_{2} \mathrm{O}$ for $10 \mathrm{~min}$, the sample was stained with hematoxylin as a counterstain and dehydration, clearing and mounting was then performed. A pixel value represented the expression intensity of the SLPI protein in the wound area and re-epithelialization. The Mac-3 positive cells were counted in the wound area. A pixel value and number of macrophages were quantified and calculated using AxioVision LE release 4.6 software (Carl Zeiss).

Gelatin zymography. Gelatin zymography was used to identify MMP-2 and MMP-9 in the tissues. Total protein (30 $\mu \mathrm{l})$ of the tissues and HT-1080 cells was electrophoresed on $8 \%$ SDSzymogram gel (Invitrogen, USA). The conditioned media from the HT-1080 human fibrosarcoma cells were loaded as a size marker for MMP-2 and MMP-9. After electrophoresis, the gels were incubated three times in a zymogram renaturing buffer (Invitrogen) at room temperature for $20 \mathrm{~min}$, and then in a zymogram developing buffer (Invitrogen) for $30 \mathrm{~min}$. The gels were then incubated at $37^{\circ} \mathrm{C}$ for $16 \mathrm{~h}$ after being transferred to the same developing buffer. After developing, the gels were stained with $0.2 \%$ Coomassie Brilliant Blue (Bio-Rad Laboratories) for $1 \mathrm{~h}$ and incubated with a destaining solution (10\% acetic acid, $40 \%$ methanol in distilled water) for $30 \mathrm{~min}$. The density of the MMP-2 and MMP-9 bands was measured using a Science Lab Image Gauge (FujiFilm).

Statistical analysis. All experiments were carried out at least in triplicate. The data is reported as the mean and standard deviation determined using the Excel 2007 statistical software (Microsoft, USA). Significant differences $\left({ }^{*} \mathrm{P}<0.05\right)$ were determined using a Student's t-test.

\section{Results}

Estrogen concentration, wound area, and migration of keratinocyte. Estrogen concentration in the peripheral blood of the PCOS group was 0.8 times lower than that in the normal group (Fig. 1A). H\&E staining was used to examine the wound healing area in the normal and PCOS groups after wounding on Days 1, 3, 5 and 7. The stained wound tissues showed that the PCOS wound area was wider than that of the normal group until Day 7 (Fig. 1B). The wound area of the PCOS group was $1.7,1.2,1.1$ and 1.7 times higher than that of the normal group on Days 1, 3, 5 and 7, respectively (Fig. 1C). The rate of keratinocyte migration in the PCOS group was 0.5 and 0.1 times lower than that in the normal group on Days 1 and 3, respectively, after wounding. The migration rate on Day 5 was similar in the two groups (Fig. 1D).

Inflammatory cells and infiltrated macrophage. Differences between the normal and PCOS group in the number of inflammatory cells recruited in the wound area were observed in the Giemsa-stained tissues (Fig. 2A). The number of inflammatory cells recruited in the PCOS group was 1.2, 1.3, 1.3 and 1.6 times higher than that of the normal group on Days 1, 3,5 and 7, respectively (Fig. 2B). Immunohistochemical staining was performed using the Mac-3 antibody to detect macrophages in the wound area (Fig. 2C). The macrophages in the wound 
B

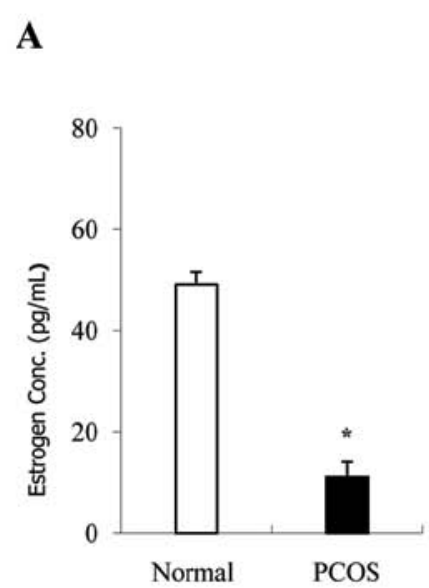

B

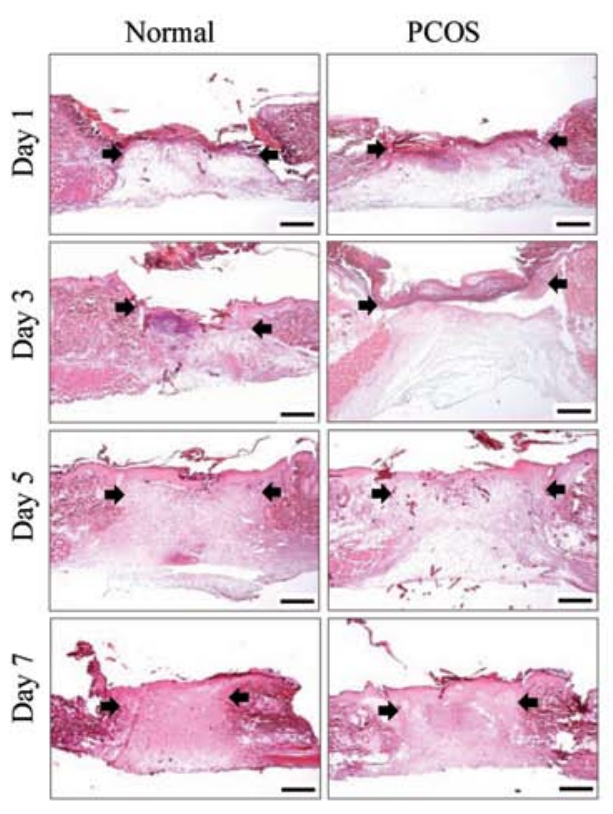

C

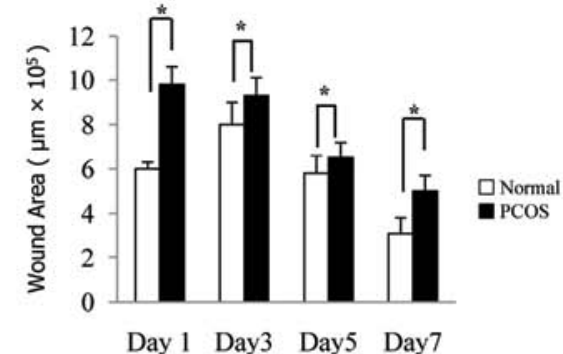

D

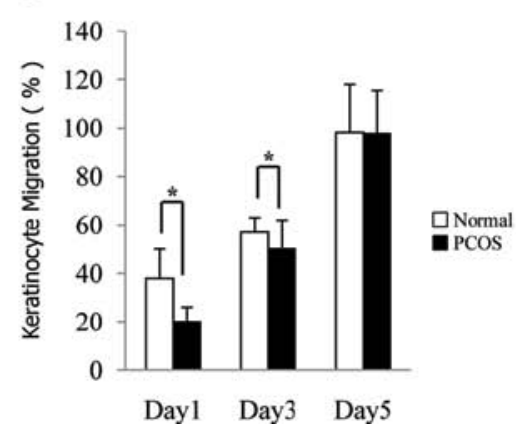

Figure 1. Estrogen concentration and differences in the skin wound area between the normal and PCOS groups. (A) The estrogen concentration in the peripheral blood of the PCOS group is lower than that in the normal group. (B) Differences in the wound area between the normal and PCOS group at the skin wound. The wound area of the PCOS group was wider than that of the normal group. (C) Morphometric analysis from the wound area in the normal and PCOS group. The wound area of the PCOS group is larger than that of the normal group from Days 1-7. (D) The keratinocyte migration rate in the PCOS group is lower than that in the normal group. Scale bars, $200 \mu \mathrm{m}$. The arrows demarcate the wound edge.

A

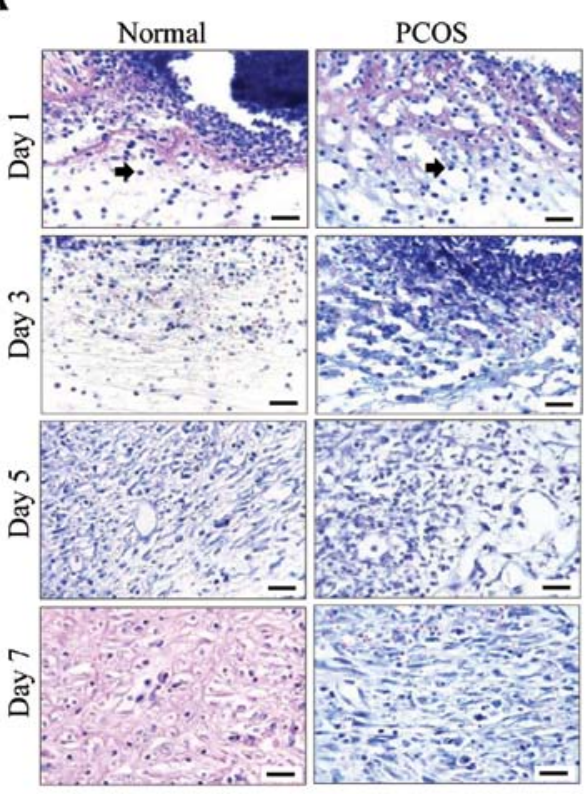

B

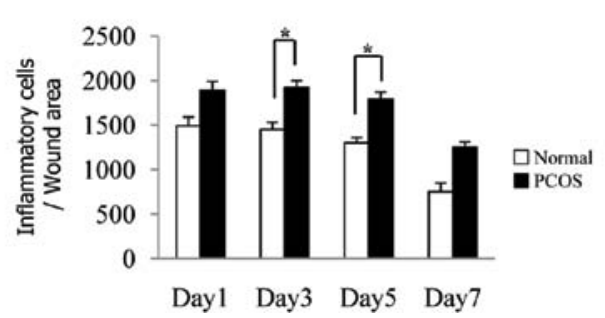

C

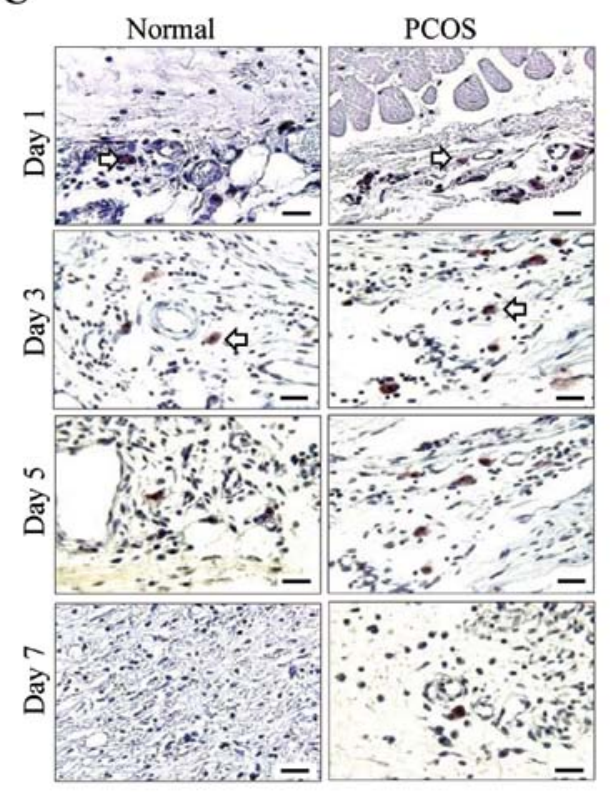

D

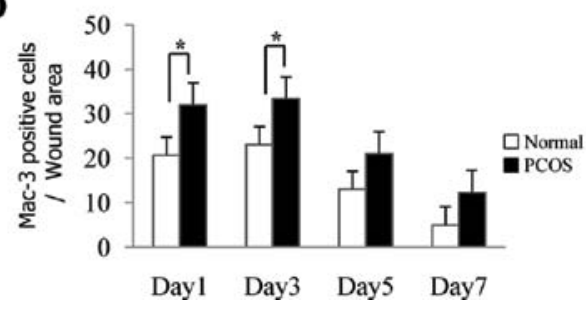

Figure 2. Infiltrated inflammatory cells and macrophages in the wound area of the normal and PCOS group. (A and B) The number of inflammatory cells in the PCOS wound area increased from Days 1-7 compared to that of the normal group. All tissues were stained with Giemsa. (C and D) The number of Mac-3 positive cells was high on Days 1 and 3 in the PCOS wound and decreased on Days 5 and 7 in all groups. Scale bars, $20 \mu \mathrm{m}$. The black and open arrows indicate inflammatory cell and macrophage, respectively. 
A

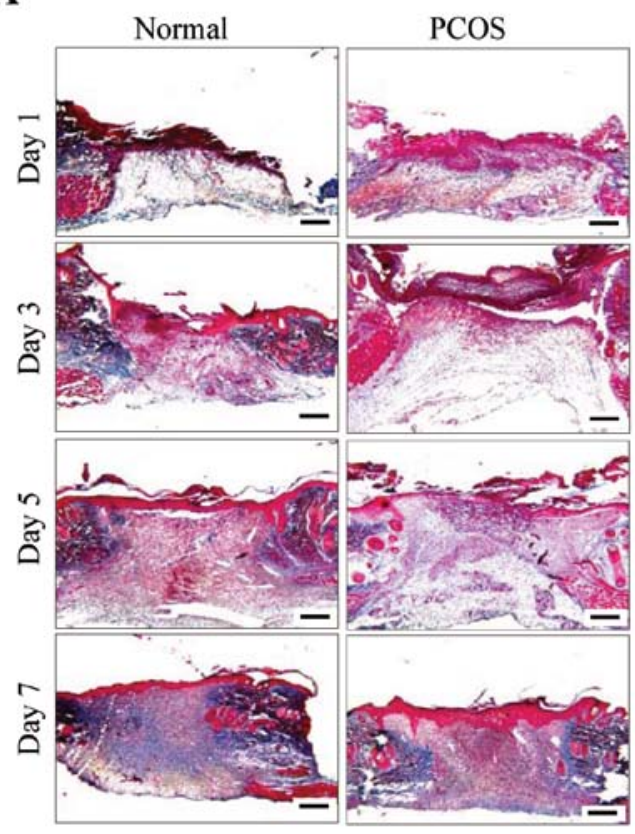

B

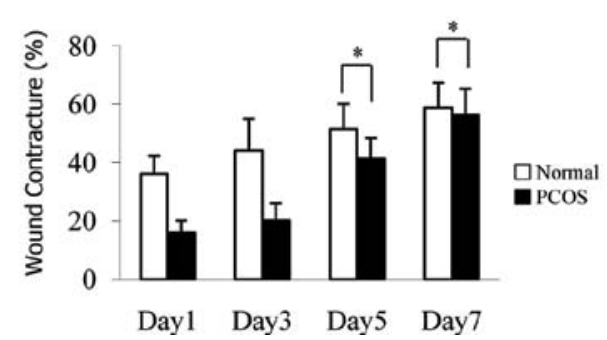

C

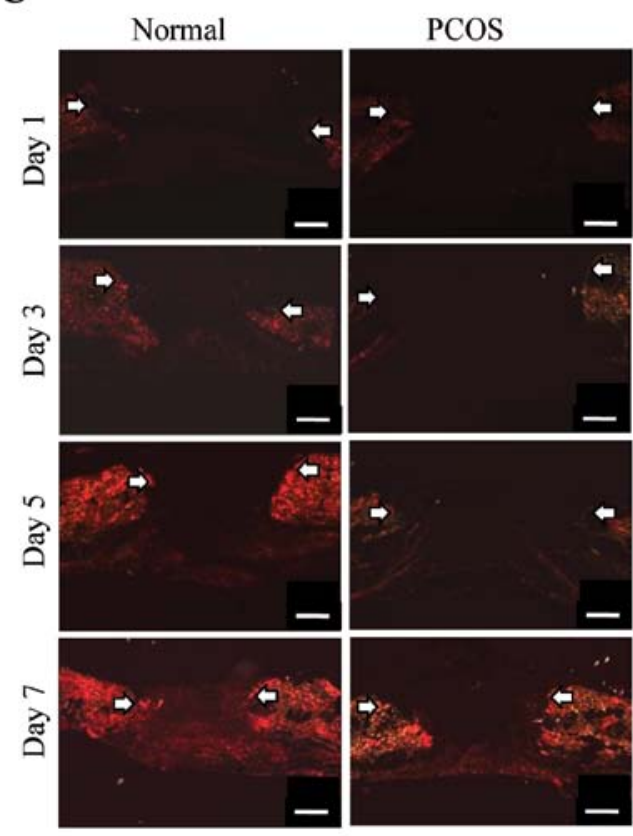

D

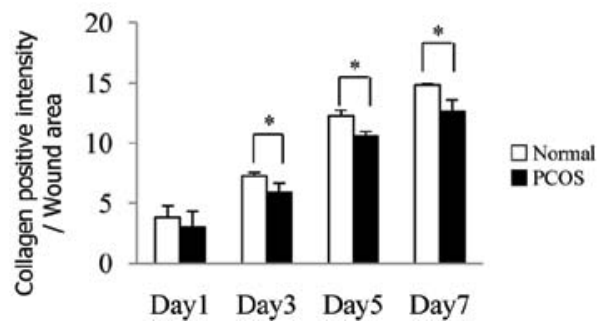

Figure 3. Accumulated collagen between normal and PCOS skin wound. (A) Differences in accumulated collagen (blue color) in the healing area between the normal and PCOS group at the skin wound. (B) Wound contracture of the PCOS group is lower than normal after wounding. (C) Differences in accumulated collagen (bright red color) in the healing area between the normal and PCOS groups on the skin wound after Picrocirius Red staining. This result coincides with that of Masson's Trichrome staining. (D) Collagen positive intensity in the wound area is low in the PCOS group from Days 1-7. Scale bars, $100 \mu \mathrm{m}$. The white arrows demarcate the wound edge.

areas of the PCOS group were 1.6 and 1.4 times higher than that of the normal group on Days 1 and 3, respectively. In addition, the number of infiltrated macrophages were lower in all groups on Days 5 and 7 (Fig. 2D).

Collagen deposition and wound contracture. Newly formed collagen was visualized by MT staining in the wound area. More collagen deposition was observed in the healing area of the normal group than the PCOS group from Days 1-7 (Fig. 3A). The wound contracture of the PCOS group was 0.2 and 0.1 times lower than that of the normal group on Days 5 and 7 after wounding, respectively (Fig. 3B). To confirm the result of MT staining, the same tissues were stained with Picrosirius Red and the level of collagen accumulation was quantified (Fig. 3C). The intensity of collagen deposited in the normal group was 1.2, 1.4, 1.2 and 1.2 times higher than that of the PCOS group on Days 1, 3, 5 and 7, respectively (Fig. 3D).

Differential expression of SLPI $m R N A$ and protein. RT-PCR and Western blotting were performed to confirm the previous immunohistochemistry result. In the unwounded tissue (control), the expression of SLPI protein was 4.3 times higher in the skin of the PCOS group than that in the normal group.
Moreover, after wounding, the mRNA and protein expression of SLPI were higher in the PCOS group than in the normal group from Days 1-7 (Fig. 4A and C). The expression intensity of SLPI mRNA in the PCOS group was 1.46 and 3.3 times higher than the normal group on Days 3 and 5, respectively. In addition, the level of the SLPI protein in the PCOS group was 3.2, 1.1 and 1.2 times higher than that in the normal group on Days 1, 3 and 5, respectively (Fig. 4B and D).

Expression of SLPI protein in the wound area and re-epithelization layer. Immunohistochemistry was performed for SLPI protein expression in the wound tissues and the intensity was measured on the epithelium or dermis of the wound area (Fig. 5A). The expression intensity of SLPI in the dermal area of the PCOS group was 1.2 times higher than that in the normal group on Days 3 and 5, respectively (Fig. 5B). In the re-epithelization layer, the intensity of SLPI of the normal group was 1.4 and 1.3 times higher than PCOS on Days 3 and 5, respectively (Fig. 5C).

The expression of MMP-2 and MMP-9. To detect the MMP activity, a zymography assay was conducted in the wound tissues. Pro- and active MMP-2 and -9 were elevated in the 
A

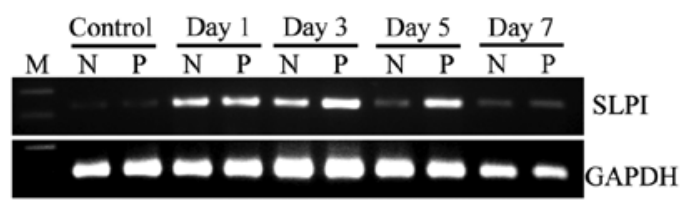

B

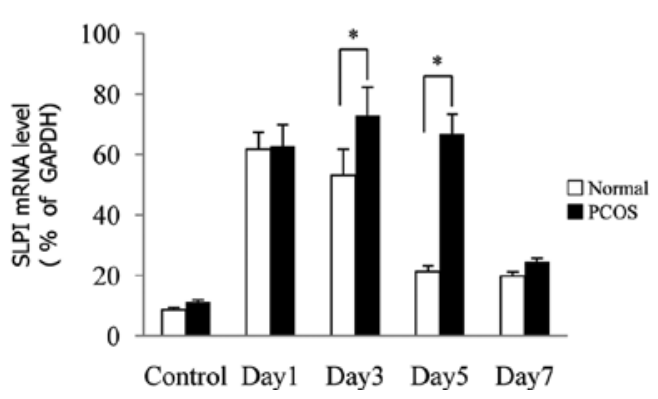

C

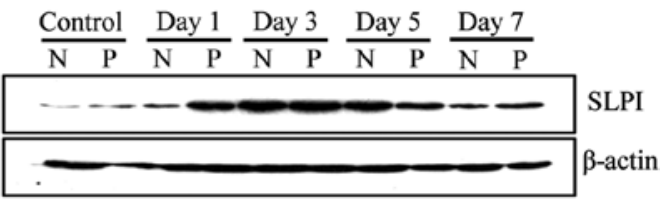

D

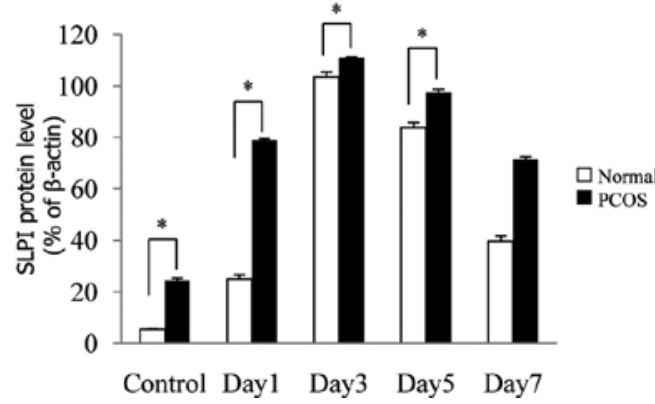

Figure 4. Differential expression of SLPI in the skin wound between the normal and PCOS groups. (A and C) SLPI mRNA and protein expression in the normal and PCOS skin wound. (B and D) Increased SLPI mRNA and protein expression in the PCOS group compared to the normal group. SLPI mRNA and protein normalized to the corresponding GAPDH and $\beta$-actin, respectively. M, size marker; N, normal; P, PCOS.

A

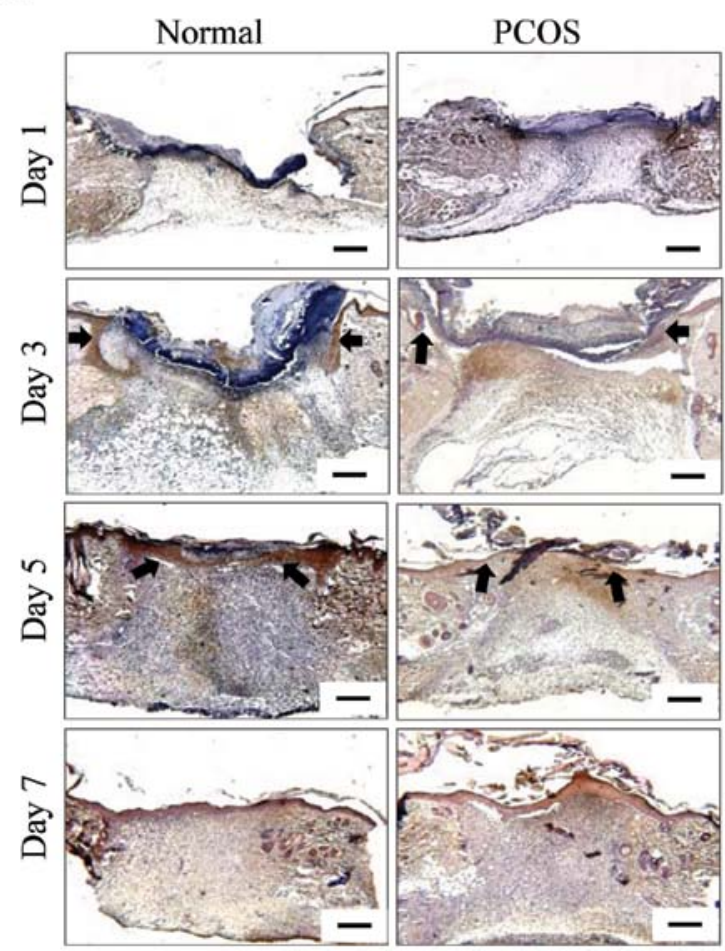

B

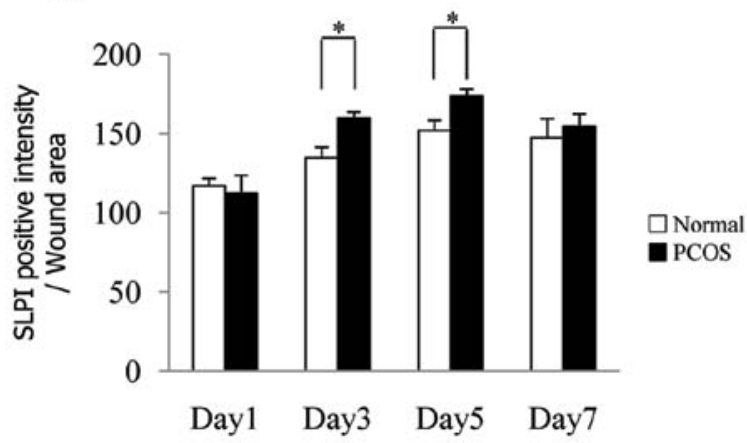

C

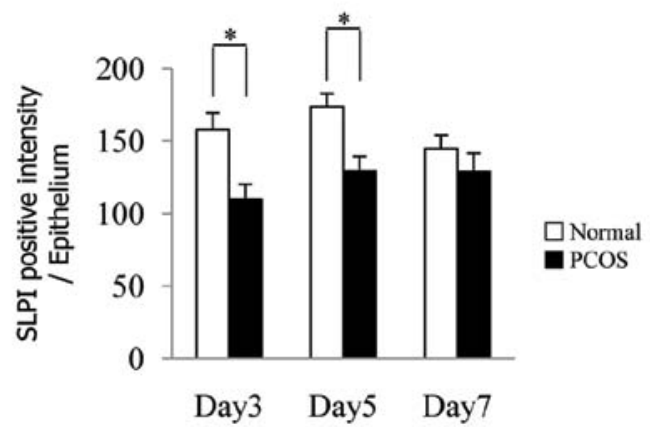

Figure 5. Immunohistochemistry for SLPI expression on the skin wound of the normal and PCOS groups. (A) Expression of the SLPI protein in the normal and PCOS groups with a skin wound were compared. (B and C) The intensity of SLPI expression was measured in the dermal wound area and epithelium. Scale bars, $200 \mu \mathrm{m}$. The black arrows indicate regenerated epithelial tongue.

normal and PCOS wound compared to that of the unwounded tissue. The MMP-9 level increased until Day 3 and decreased on Days 5 and 7 but the MMP-2 level increased gradually after wounding (Fig. 6A). Active MMP-2 and -9 in the PCOS group were significantly lower than that of the normal group until Day 5 but the active forms in PCOS were higher than in the normal group on Day 7 (Fig. 6B and C).
The expression of TGF- $\beta 1 \mathrm{mRNA}$ and protein. In the unwounded tissue (control), the level of TGF- $\beta 1$ expression was lower in the skin of the PCOS group than in the normal group. TGF- $\beta 1$ was elevated in the normal and PCOS wound compared to that of the unwounded tissue. After wounding, the increased mRNA and protein expression of TGF- $\beta 1$ was lower in the PCOS group than that of the normal group from 
A

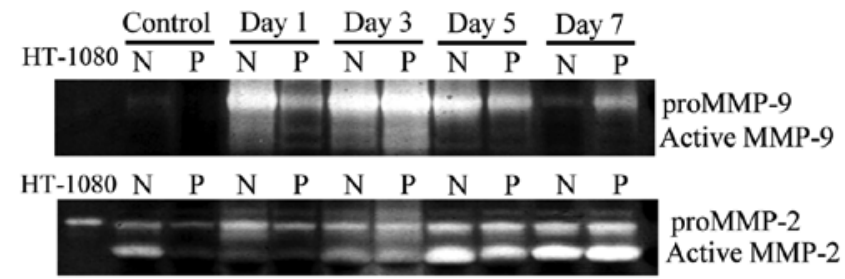

B

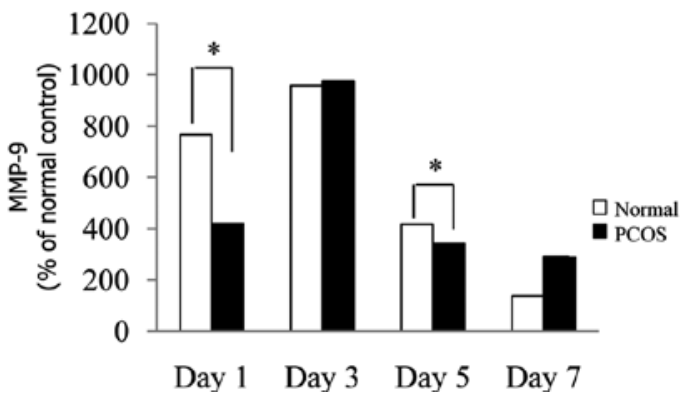

C

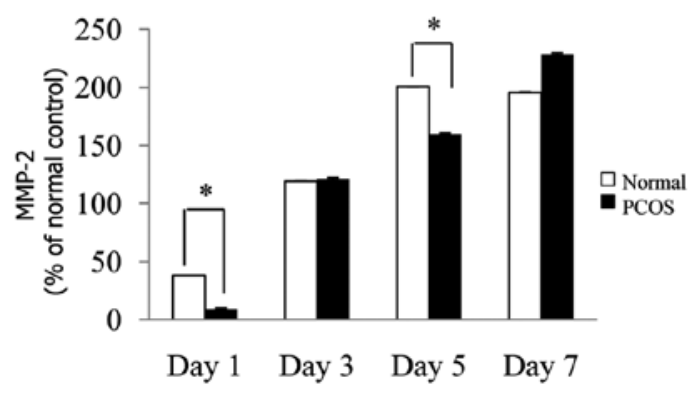

Figure 6. Differential expression of MMP-2 and MMP-9 in skin wounds between the normal and PCOS group. (A) Pro- and active MMP-2 and -9 were detected by zymography. (B and C) Active MMP-2 and -9 in PCOS group were significantly lower than that of the normal group. N, normal; P, PCOS.

A

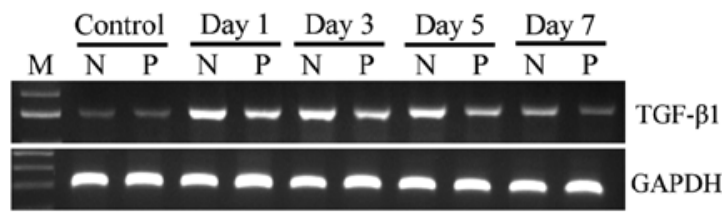

B

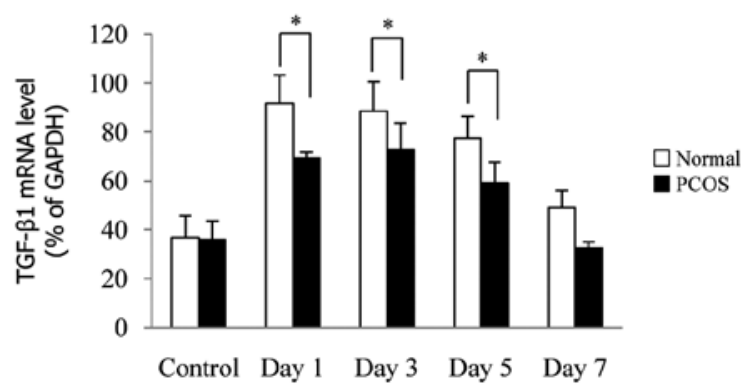

C

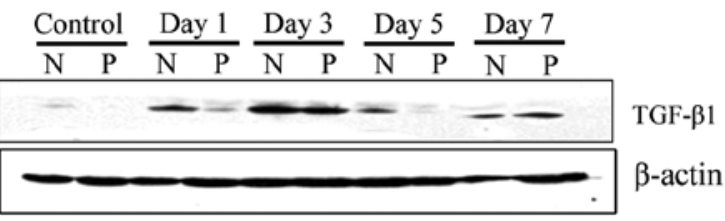

D

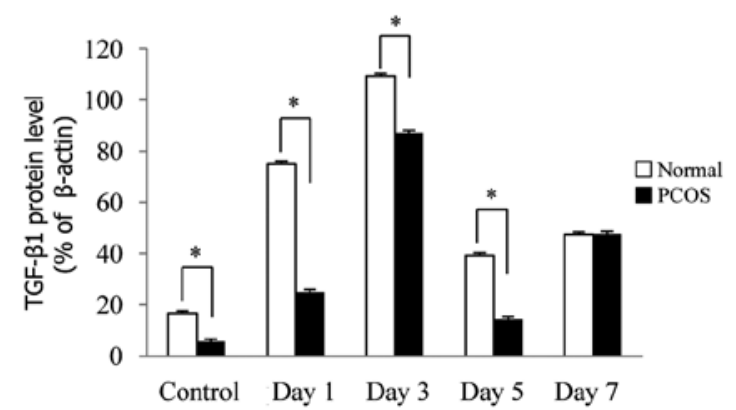

Figure 7. Expression of TGF- $\beta 1$ in the skin wound between the normal and PCOS group. (A and C) TGF- $\beta 1$ mRNA and protein expression in the normal and PCOS skin wounds. (B and D) Low TGF- $\beta 1 \mathrm{mRNA}$ expression and protein levels in the PCOS skin wound compared to the normal group. TGF- $\beta$ mRNA and protein were normalized to the corresponding GAPDH and $\beta$-actin, respectively. M, size marker; N, normal; P, PCOS.

Days 1-7 (Fig. 7A and C). The expression intensity of TGF- $\beta 1$ mRNA in the PCOS group was 0.2 and 0.2 times lower than that of the normal group on Days 1 and 3, respectively. The level of the TGF- $\beta 1$ protein in the PCOS group was 0.7, 0.2 and 0.7 times lower than that of the normal group on Days 1, 3 and 5, respectively (Fig. 7B and D).

\section{Discussion}

Estrogen plays an important role in skin aging, which is a delay in the thinning of the epithelium and preservation of skin thickness and moisture through recovery of the decreased collagen content and elasticity in the skin in postmenopausal women $(24,25)$. EV is one of the synthetic estrogens used for hormone therapy. Excessive administration of EV rapidly leads to a decrease in the estrogen concentration in the blood, which induces PCOS (2), consistent with the result of the blood estrogen level after establishment of a PCOS rat with EV. A decrease in the estrogen level in OVX mice induces delayed wound healing, such as an extension of the wound area, excessive inflammatory response, retardation of re-epithelization and collagen deposition (8). The skin wound area of the SLPI 
null mice was larger than that of the normal mice and wound healing was delayed in the oral mucosa and skin $(19,20)$. In the OVX rat, SLPI was not expressed in the uterine tissues but increased after estrogen treatment (22). When the human uterine epithelial cells (UECs) were incubated with estrogen, the secretion of SLPI was enhanced but the secretion of inflammatory cytokines, such as interleukin-6 (IL-6) and IL-8, and decreased after stimulation with lipopolysaccharide (LPS) (26). These results show that the inhibitory function of estrogen to inflammatory cytokines is mediated by SLPI regulation and suggest that SLPI secretion is controlled by the hormone, estrogen, but not progesterone in human UECs. In this study, delayed healing in PCOS rat skin wound is consistent with the results showing a large wound area, slow re-epithelization, increased inflammation and matrix degradation. Interestingly, SLPI mRNA and protein expression was higher in the PCOS rat skin than the normal rat skin, and also increased in the PCOS group after wounding, which is consistent with our previous results of SLPI induction in a polycystic ovary (4). Recently, microarray and qPCR data for estrogen-regulated gene expression in old and young human subjects demonstrated that SLPI expression is higher in old human skin than in young human normal skin, which is consistent with the present result (27) and the unpublished results for SLPI expression in OVX mouse normal skin (data not shown). Therefore, an increase in SLPI expression caused by a decrease in estrogen might inhibit the disruption of tissue in the PCOS rat skin. Although estrogen is considered to be one of the precursor molecules to induce and regulate SLPI expression, SLPI may play an important role on the effects of estrogen in the PCOS rat skin wound healing process.

Estrogen promotes re-epithelization by stimulation of keratinocyte proliferation during wound healing in mouse skin $(8,28)$. Estrogen receptor $\beta(E R \beta)$ is strongly expressed in the human stratum basal and spinosum of the scalp epithelium (29) and the increase in ER $\alpha$ expression is induced by estrogen in keratinocytes (30). SLPI regulates the proliferation of human endometrial epithelial cells and is expressed strongly to promote the proliferation of carcinoma cells $(16,17,31)$. In addition, re-epithelization was delayed in the SLPI knockout mice skin wound healing model (19). In this study, the rate of keratinocyte migration was lower in the PCOS skin wound than in the normal group. In particular, SLPI protein expression by immunohistochemistry was also low in the regenerated epithelium of a PCOS skin wound and was different from the dermal expression. Therefore, the decreased estrogen level in the PCOS rat skin induces delayed re-epithelization during the wound healing process with decreasing SLPI expression in the epithelial cells, suggesting that a decrease in SLPI expression in keratinocytes may be regulated by estrogen during epithelial cell proliferation and migration in PCOS skin wounds.

Estrogen accelerates cutaneous wound healing by a decrease in the local inflammatory response through the inhibition of MIF expression and decreases the infiltration of macrophages in a skin wound of OVX mice $(32,33)$. Estrogen prevents excessive extracellular matrix degradation by elastase through the inhibition of neutrophil migration (11) while TGF- $\beta 1$ induces rapid chemotaxis of neutrophils and monocytes into the wound site (34). SLPI is expressed in inflammatory cells, such as mast cells, neutrophils and macrophages (35) and reduces the levels of TNF- $\alpha$ and macrophage inflammatory protein-1 (MIP-1) secretion in damaged lung and liver, resulting in the prevention of neutrophil migration (36). The topical treatment of SLPI inhibits the chymase activity secreted from mast cells at the site of conjunctivitis, which interrupts the infiltration of polymorphonuclear leukocytes and monocytes (37).

In SLPI deficient mice, the infiltration of neutrophils and macrophages increased in the oral wound area (20). Therefore, a decrease in estrogen concentration or an absence of SLPI causes an excessive inflammatory response and delayed matrix remodeling due to the increased infiltration of neutrophils, and inordinate concentration of protease. In the present study, the number of inflammatory cells and macrophages was high from Days 1-7 in the wound area of PCOS compared to the normal group consistent with a previous report. Moreover, although SLPI expression was higher in the PCOS wound area, SLPI could not reduce the number of infiltrated inflammatory cells. A previous study demonstrated that estrogen affects skin wound healing by regulating the inflammatory response, cytokine expression and matrix deposition, suggesting that estrogen systematically regulates many cytokines in the inflammatory response (38) in conjunction with a low level of TGF- $\beta 1$ expression in a PCOS rat skin wound. Therefore, a modified inflammatory cellular response by a change in estrogen may not be affected by the induction of SLPI in PCOS rat skin wound.

The collagen content of the skin in postmenopausal women decreases by $1-2 \%$ per year but estrogen has an effect on the recovery of collagen (39). Estrogen consequently increases collagen and fibronectin in wound areas (11) and might improve wound healing by reducing MMP-mediated collagenolysis (40). On the other hand, systemic estrogen treatments do not alter the amount of collagen or rate of collagen synthesis in postmenopausal women (41). OVX rat wound healing showed a decrease in collagen deposition (32). MMP-2 and -9 were activated significantly by the sera from PCOS women compared to that from normal women (42). In the present study, collagen deposition in a wound area was low in the PCOS compared to the normal skin during the healing period despite the high level of SLPI mRNA and protein expression in a PCOS skin wound. Interestingly, with increasing SLPI expression, MMP-2 and -9 expression was lower in both the unwounded and wounded PCOS skin than in the normal skin. In our previous results, SLPI and MMP-2 expression was high and low in polycystic ovaries compared to normal ovaries suggesting that SLPI might act as a protease inhibitor rather than an anti-inflammatory cytokine in the PCOS rat ovary (4). In the skin wounds of SLPI-deficient mice, the level of MMP-2 and -9 secretion was higher than that in a normal mice wound (20). In bronchial epithelial cells, the expression of MMP-9 and IL-8 increased during the inflammatory response but decreased after SLPI treatment (43). It is surprising that the effects of estrogen not on SLPI but on the expression of TGF- $\beta 1$ explained this result. The expression of TGF- $\beta 1$ mRNA and protein was low in the PCOS rat skin than in the normal control group, and it showed a low level in the PCOS group after wounding. Estrogen increases the level of collagen synthesis and inhibits the degradation of the extracellular matrix by promoting the secretion of TGF- $\beta 1$ on dermal fibroblasts (8-10). Estrogen also increases the level of TGF- $\beta 1$ 
in osteoblasts (44) and ovariectomy down-regulates TGF- $\beta 1$ transcription in the bone (45) while SLPI reduces the TGF- $\beta 1$ activity (19), suggesting that estrogen and SLPI may regulate the production of TGF- $\beta 1$ in PCOS skin wounds. Therefore, the delayed matrix deposition in a PCOS skin wound was attributed to the low content of collagen by the low TGF- $\beta 1$ and high SLPI expression compared to that of a normal skin wound, which is consistent with the results of MT and Picrosirius Red staining, suggesting that the low expression of TGF- $\beta 1$ by estrogen and SLPI may lead to lower collagen deposition in a PCOS skin wound.

Overall, this is the first report of the PCOS skin wound healing process and induction of SLPI in PCOS skin wounds. The expression of SLPI was higher in the unwounded skin of the PCOS group than in the normal skin and was also higher in the wounded skin of the PCOS compared to the normal subjects. Although SLPI expression was up-regulated, the healing process was delayed in the PCOS skin wound. In view of previous results, the increased SLPI in the PCOS skin wound is associated with the prevention of an excessive inflammatory response and aberrant collagen deposition but it is not enough to accelerate PCOS skin wound healing. This suggests that SLPI may act as a local protease inhibitor rather than a systemic modulating molecule in the PCOS rat skin wound. Previous studies suggested that SLPI may act as a molecule for hormonal replacement therapy, but they did not demonstrate the function and expression of SLPI in a PCOS skin wound. Therefore, although SLPI did not completely compensate for the role of estrogen in PCOS rat skin wound healing, it may play an important role independently of estrogen in skin wounds, such as PCOS, which has a hormonal imbalance by a reduced estrogen level. This suggests that SLPI may be a potential agent for hormonal replacement treatment on the chronic inflammation and wound healing of postmenopausal women. Future studies will need to address this possibility by evaluating the effects of SLPI by itself and/or of the neutralization of SLPI with antibody.

\section{Acknowledgements}

This study was supported by the Korea Research Foundation (KRF-2006-311-E00171) and the National Research Foundation of Korea (NRF) funded by the Ministry of Education, Science and Technology (no. R13-2008-010-00000-0).

\section{References}

1. Werner S, Krieg T and Smola H: Keratinocyte-fibroblast interactions in wound healing. J Invest Dermatol 127: 998-1008, 2007.

2. Schulster A, Farookhi R and Brawer JR: Polycystic ovarian condition in estradiol valerate-treated rats: spontaneous changes in characteristic endocrine features. Biol Reprod 31: 587-593, 1984.

3. Brawer JR, Munoz M and Farookhi R: Development of the polycystic ovarian condition (PCO) in the estradiol valerate-treated rat. Biol Reprod 35: 647-655, 1986.

4. Park JJ, Bae CS, Choi BD, et al: Induction of secretory leukocyte protease inhibitor (SLPI) in estradiol valerate (EV) induced polycystic ovary. Arch Pharm Res 34: 1389-1397, 2011.

5. Balen AH: The pathogenesis of polycystic ovary syndrome: the enigma unravels. Lancet 354: 966-967, 1999.

6. Koninckx PR, Lauweryns JM and Cornillie FJ: Endometrial effects during hormone replacement therapy with a sequential oestradiol valerate/cyproterone acetate preparation. Maturitas 16: 97-110, 1993.
7. Mowad CM, Margolis DJ, Halpern AC, Suri B, Synnestvedt M and Guzzo CA: Hormonal influences on women with psoriasis. Cutis 61: 257-260, 1998

8. Ashcroft GS, Dodsworth J, van Boxtel E, et al: Estrogen accelerates cutaneous wound healing associated with an increase in TGF- $\beta 1$ levels. Nat Med 3: 1209-1215, 1997.

9. Grande JP: Role of TGF- $\beta$ in tissue injury and repair. Proc Soc Exp Biol Med 214: 27-40, 1997.

10. Beck LS, DeGuzman L, Lee WP, Xu Y, Siegel MW and Amento EP: One systemic administration of transforming growth factor- $\beta 1$ reverses age- or glucocorticoid-impaired wound healing. J Clin Invest 92: 2841-2849, 1993.

11. Ashcroft GS, Greenwell-Wild T, Horan MA, Wahl SM and Ferguson MW: Topical estrogen accelerates cutaneous wound healing in aged humans associated with an altered inflammatory response. Am J Pathol 155: 1137-1146, 1999.

12. Thompson RC and Ohlsson K: Isolation, properties, and complete amino acid sequence of human secretory leukocyte protease inhibitor, a potent inhibitor of leukocyte elastase. Proc Natl Acad Sci USA 83: 6692-6696, 1986.

13. Eisenberg SP, Hale KK, Heimdal P and Thompson RC: Location of the protease-inhibitory region of secretory leukocyte protease inhibitor. J Biol Chem 265: 7976-7981, 1990.

14. Choi BD, Jeong SJ, Wang G, Kim HJ, Kim BO, Hwang HK, Lim DS, Kim SH and Jeong MJ: Temporal induction of secretory leukocyte protease inhibitor (SLPI) in odontoblasts by lipopolysaccharide and wound infection. J Endod 35: 997-1002, 2009.

15. Sallenave JM: Antimicrobial activity of antiproteinases. Biochem Soc Trans 30: 111-115, 2002.

16. Choi BD, Jeong SJ, Wang G, et al: Secretory leukocyte protease inhibitor is associated with MMP-2 and MMP-9 to promote migration and invasion in SNU638 gastric cancer cells. Int J Mol Med 28: 527-534, 2011.

17. Wang G, Lim DS, Choi BD, et al: Effect of secretory leukocyte protease inhibitor on migration and invasion of human KB oral carcinoma cells. Animal Cells System 15: 139-146, 2011.

18. Holcomb VB, Keck VA, Barrett JC, Hong J, Libutti SK and Nunez NP: Obesity impairs wound healing in ovariectomized female mice. In Vivo 23: 515-518, 2009.

19. Ashcroft GS, Lei K, Jin W, et al: Secretory leukocyte protease inhibitor mediates non-redundant functions necessary for normal wound healing. Nat Med 6: 1147-1153, 2000.

20. Angelov N, Moutsopoulos N, Jeong MJ, Nares S, Ashcroft G and Wahl SM: Aberrant mucosal wound repair in the absence of secretory leukocyte protease inhibitor. Thromb Haemost 92: 288-297, 2004.

21. King AE, Morgan K, Sallenave JM and Kelly RW: Differential regulation of secretory leukocyte protease inhibitor and elafin by progesterone. Biochem Biophys Res Commun 310: 594-599, 2003.

22. Chen D, Xu X, Cheon YP, Bagchi MK and Bagchi IC: Estrogen induces expression of secretory leukocyte protease inhibitor in rat uterus. Biol Reprod 71: 508-514, 2004.

23. Kwon KB, Lim YK, Choi MJ and Ahn RS: Spontaneous maturation of follicular oocyte in Rana dybowskii in vitro: seasonal influences, progesterone production and involvement of cAMP. J Exp Zool 252: 190-199, 1989.

24. Varila E, Rantala I, Oikarinen A, et al: The effect of topical estradiol on skin collagen of postmenopausal women. Br J Obstet Gynaecol 102: 985-989, 1995.

25. Sumino H, Ichikawa S, Abe M, Endo Y, Ishikawa $\mathrm{O}$ and Kurabayashi M: Effects of aging, menopause, and hormone replacement therapy on forearm skin elasticity in women. J Am Geriatr Soc 52: 945-949, 2004.

26. Fahey JV, Wright JA, Shen L, Smith JM, Ghosh M, Rossoll RM, and Wira CR: Estradiol selectively regulates innate immune function by polarized human uterine epithelial cells in culture. Mucosal Immunol 1: 317-325, 2008.

27. Hardman MJ and Ashcroft GS: Estrogen, not intrinsic aging, is the major regulator of delayed human wound healing in the elderly. Genome Biol 9: 1-17, 2008.

28. Kanda $\mathrm{N}$ and Watanabe S: $17 \beta$-estradiol stimulates the growth of human keratinocytes by inducing cyclin D2 expression. J Invest Dermatol 123: 319-328, 2004.

29. Thornton MJ, Taylor AH, Mulligan K, et al: Estrogen receptor beta $(E R \beta)$ is the predominant estrogen receptor in human scalp. Exp Dermatol 12: 181-190, 2003.

30. Verdier-Sevrain S, Yaar M, Cantatore J, Traish A and Gilchrest BA: Estradiol induces proliferation of keratinocytes via a receptor mediated mechanism. FASEB J 18: 1252-1254, 2004. 
31. Zhang D, Simmen RC, Michel FJ, Zhao G, Vale-Cruz D and Simmen FA: Secretory leukocyte protease inhibitor mediates proliferation of human endometrial epithelial cells by positive and negative regulation of growth associated genes. J Biol Chem 277: 29999-30009, 2002.

32. Ashcroft GS, Mills SJ, Lei K, et al: Estrogen modulates cutaneous wound healing by downregulating macrophage migration inhibitory factor. J Clin Invest 111: 1309-1318, 2003.

33. Hardman MJ, Emmerson E, Campbell L and Ashcroft GS Selective estrogen receptor modulators accelerate cutaneous wound healing in ovariectomized female mice. Endocrinology 149: 551-557, 2008.

34. Wahl SM, Hunt DA, Wakefield L, et al: Transforming growth factor type beta induces monocyte chemotaxis and growth factor production. Proc Natl Acad Sci USA 84: 5788-5792, 1987.

35. Mihaila A and Tremblay GM: Human alveolar macrophages express elafin and secretory leukocyte protease inhibitor. Z Naturforsch 56: 291-297, 2001

36. Lentsch AB, Yoshidome H, Warner RL, Ward PA and Edwards MJ: Secretory leukocyte protease inhibitor in mice regulates local and remote organ inflammatory injury induced by hepatic ischemia/reperfusion. Gastroenterology 117: 953-961, 1999.

37. Murata E, Sharmin S, Shiota H, Shiota M, Yano M and Kido H: The effect of topically applied secretory leukocyte protease inhibitor on the eosinophil response in the late phase of allergic conjunctivitis. Curr Eye Res 26: 271-276, 2003.

38. Gilliver SC, Ashworth JJ and Ashcroft GS: The hormonal regulation of cutaneous wound healing. Clin Dermatol 25: 56-62, 2007.
39. Brincat M, Versi E, Moniz F, Magos A, de Trafford J and Studd JW: Skin collagen changes in postmenopausal women receiving different regimens of estrogen therapy. Obstet Gynecol 70: 123-127, 1987.

40. Pirila E, Ramamurthy N, Maisi P, et al: Wound healing in ovariectomized rats: effects of chemically modified tetracycline (CMT-8) and estrogen on matrix metalloproteinases $-8,-13$ and type 1 collagen expression. Curr Med Chem 8: 281-294, 2001.

41. Haapasaari KM, Raudaskoski T, Kallioinen M, et al: Systemic therapy with estrogen or estrogen with progestin has no effect on skin collagen in postmenopausal women. Maturitas 27: 153-162, 1997.

42. Tan BK, Adya R, Chen J, Lehnert H, Cassia LJS and Randeva HS Metformin treatment exerts antiinvasive and antimetastatic effects in human endometrial carcinoma cells. J Clin Endocrinol Metab 96: 808-816, 2011.

43. Qu MY, Luo BL, Chen HM, Feng JT and Gu QH: Effects of secretory leukocyte protease inhibitor on expression of inflammation mediators in normal human bronchial epithelial cells induced by cigarette smoke extract. Zhonghua Jie He He Hu Xi Za Zhi 31: 352-355, 2008.

44. Oursler MJ, Cortese C, Keeting P, et al: Modulation of transforming growth factor- $\beta$ production in normal human osteoblast-like cells by $17 \beta$-estradiol and parathyroid hormone. Endocrinology 129: 3313-3320, 1991

45. Ikeda T, Shigeno C, Kasai R, et al: Ovariectomy decreases the mRNA levels of transforming growth factor-beta 1 and increases the mRNA levels of osteocalcin in rat bone in vivo. Biochem Biophys Res Commun 194: 1228-1233, 1993. 